\title{
FACE: the barefaced facts of Al potency
}

This article was published in the following Dove Press journal:

Cancer Management and Research

23 October 2010

Number of times this article has been viewed

\section{Alain Monnier \\ Institut Régional Fédératif du Cancer (IFRC), Centre Hospitalier Belfort- Montbéliard, Montbeliard Cedex, France}

\begin{abstract}
The use of third-generation aromatase inhibitors (AIs), such as anastrozole and letrozole, as initial adjuvant hormonal therapy in postmenopausal women (PMW) with hormone receptor-positive $(\mathrm{HR}+)$ breast cancer offers a significant benefit over tamoxifen for reducing recurrence risk. Clinical studies, including the Arimidex Tamoxifen Alone or in Combination (ATAC) and the Breast International Group (BIG) 1-98 trials, have proven that both anastrozole and letrozole are, respectively, superior to tamoxifen in improving disease-free survival. Although differing in design, objectives, and follow-up time, these trials offer some insight into the comparative clinical efficacy of these two nonsteroidal AIs. In particular, results from BIG 1-98 show that letrozole significantly reduces early distant metastatic (DM) events, which constitute the majority of early recurrence events. Subsequently, there is a beneficial overall survival effect emerging in the trial, whereas survival is unchanged with anastrozole after 100 months of follow-up in ATAC. Significant differences in the potency of these two drugs, vis-à-vis their degree of aromatase inhibition, have been observed in comparative trials and show that letrozole causes a more complete suppression of estrogen levels than does anastrozole. Whether this difference in potency is relevant to reductions in DM events during adjuvant therapy remains unclear. The Femara Anastrozole Clinical Evaluation trial is addressing this issue in a more unequivocal manner by comparing initial adjuvant treatment with anastrozole or letrozole in a population of breast cancer patients at high risk of recurrence: PMW with HR+ disease and axillary lymph node involvement.
\end{abstract}

Keywords: anastrozole, aromatase inhibitors, hormone receptor-positive $(\mathrm{HR}+)$ breast cancer, letrozole, postmenopausal women, tamoxifen

\section{Introduction}

Adjuvant hormonal therapy remains an integral component of treatment for patients with hormone receptor-positive $(\mathrm{HR}+)$ early breast cancer, and antagonism of estrogen $\left(\mathrm{E}_{2}\right)$ has been the foundation of treatments to reduce the risk of breast cancer recurrence. Most notable in this respect is the selective estrogen receptor (ER) modulator tamoxifen, which has been in use since the 1970s and has been proven to significantly reduce both breast cancer recurrence and death from breast cancer in patients with $\mathrm{HR}+$, successfully resected disease. ${ }^{1}$ Tamoxifen acts via a competitive inhibition of $\mathrm{E}_{2}$ binding to the $\mathrm{ER}$, thereby inhibiting the proliferative action of $\mathrm{E}_{2}$ in breast cancer cells. The drug also may act as an estrogen agonist in selected tissues such as the endometrium, and has been associated with a number of adverse effects, including uterine cancer and thromboembolic events. ${ }^{2,3}$ In postmenopausal women (PMW), although ovarian $\mathrm{E}_{2}$ production has ceased, significant plasma levels of $\mathrm{E}_{2}$ may still exist via 
peripheral conversion of androgens to $\mathrm{E}_{2}$, a process entirely dependent on the activity of the enzyme aromatase. ${ }^{4,5}$ Therefore, aromatase has been a target of breast cancer therapy for PMW, and three generations of aromatase inhibitors (AIs) have been developed over the years. ${ }^{46}$ AIs are characterized into two types: steroidal aromatase inactivators, which bind irreversibly to the androstenedione binding site of aromatase, and nonsteroidal AIs, which bind in a reversible fashion to the heme component of aromatase. ${ }^{4-6}$ First-generation nonsteroidal AIs such as aminoglutethimide became available as early as the 1970s but were of limited use because of their nonselectivity and corresponding side-effect profiles, which included adrenal insufficiency, nausea, rash, somnolence, and blood dyscrasias. ${ }^{5,6}$ The second-generation nonsteroidal drug fadrozole also was suboptimal because of its effects on adrenal and mineralocorticoid hormone suppression. ${ }^{5}$ The third-generation nonsteroidal AIs anastrozole and letrozole, as well as the steroidal aromatase inactivator exemestane, were approved for clinical use in the 1990s. All three of these drugs are highly selective for aromatase and show good oral bioavailability. 4

In major clinical studies comparing initial adjuvant therapy between the third-generation AIs anastrozole or letrozole with tamoxifen, a significant benefit of AI treatment in reducing disease recurrence and improving disease-free survival (DFS) has been observed. ${ }^{7-12}$ Another trial has examined the efficacy of a switch to exemestane after 2 to 3 years of prior treatment with tamoxifen relative to continued tamoxifen treatment (switch adjuvant therapy); this trial also showed a benefit of AI therapy versus continued tamoxifen. ${ }^{13}$ Yet another trial has examined the use of letrozole relative to placebo after about 5 years of tamoxifen therapy (extended adjuvant therapy). ${ }^{14}$ These pivotal trials have led to the approval of AIs for use in these different treatment settings (ie, initial, switch, and extended adjuvant therapy). The nonsteroidal AIs anastrozole and letrozole, in particular, have been approved for use in the initial adjuvant setting and are significantly more effective than tamoxifen in improving DFS. Both drugs are also well tolerated, with predictable adverse event profiles that closely mimic changes associated with menopause, such as bone loss and arthralgias, but with lower incidences of other serious events, such as thromboembolic and gynecologic events, compared with tamoxifen. ${ }^{8-10}$ Accordingly, it has been generally assumed that these two nonsteroidal AIs are largely interchangeable with regard to efficacy and tolerability relative to tamoxifen; however, evidence suggests greater potency with letrozole compared with anastrozole. ${ }^{15,16}$ This review discusses the evidence for a greater potency of letrozole in aromatase inhibition, the apparent clinical correlates of this inhibition as gleaned from pivotal trials, and the design and undertaking of the Femara Anastrozole Clinical Evaluation (FACE) trial, specifically designed to resolve this issue unequivocally.

\section{Initial adjuvant therapy: clinical evidence for efficacy differences between anastrozole and letrozole The Arimidex Tamoxifen Alone or in Combination (ATAC) trial}

The ATAC trial was originally designed to determine the efficacy and safety of initial adjuvant anastrozole $(n=3125)$, tamoxifen $(\mathrm{n}=3116)$, or the two agents in combination $(n=3125)$ in PMW with early breast cancer. ${ }^{17}$ The trial was funded and implemented by the manufacturer of anastrozole. At the earliest analysis (median 33-month follow-up), it was determined that the combination arm was not significantly different from tamoxifen alone, and the arm was discontinued with no further follow-up of this population of patients $(n=3125)$. DFS was defined in the trial as the time to local or distant recurrence, a new primary breast cancer, or death from any cause. ${ }^{17}$ Importantly, when the ATAC trial was initiated, hormone receptor-negative (HR-) or HR-unknown patients were not excluded from the trial; thus, only about $84 \%$ of the trial population was $\mathrm{HR}+(\mathrm{n}=5216)$. In this clinically important population, the hazard ratio (HR) for DFS was significantly greater in the anastrozole group (HR 0.78; 95\% confidence interval $[\mathrm{CI}]: 0.65-0.93 ; P=0.002) .{ }^{17} \mathrm{At}$ this first follow-up, a sufficient number of distant metastatic (DM) events had not occurred ( $n=615$ of 704 required); thus, the incidence of DM recurrence was not reported. Contralateral breast cancer (CLBC), however, was significantly reduced in the $\mathrm{HR}+$ population (odds ratio [OR] 0.29; 95\% CI: $0.13-0.64 ; P=0.002) .{ }^{17}$ The difference in deaths between anastrozole and tamoxifen was small ( $\mathrm{n}=200$ vs 203 deaths, respectively), and thus an overall survival (OS) HR was not reported.

Subsequent analysis of the ATAC trial at a median 68-month (5.7 years) follow-up confirmed the earlier findings, with a significant reduction in recurrence events in HR+ patients (Table 1). ${ }^{8}$ In this first analysis of DM events, a total of 699 had occurred $(n=324$ anastrozole vs 375 tamoxifen), and there was a significant improvement in the endpoint of time to distant recurrence (TTDR) with anastrozole relative to tamoxifen in the intent-to-treat (ITT) population (HR 0.86; 95\% CI: 0.74-0.99; $P=0.04$ ); however, in the HR+ population, TTDR was not significantly 
Table I A comparison of key efficacy outcomes in the ATAC and BIG I-98 trials ${ }^{7-12}$

\begin{tabular}{|c|c|c|c|c|c|c|}
\hline Parameters & $\begin{array}{l}\text { ATAC }^{a} \\
(\mathrm{~N}=52 \mid 6)\end{array}$ & $\begin{array}{l}\text { ATAC }^{a} \\
(N=5216)\end{array}$ & $\begin{array}{l}\text { BIG I-98 } \\
(\mathrm{N}=80 \mid 0)\end{array}$ & $\begin{array}{l}\text { BIG I-98 } \\
(\mathrm{N}=80 \mid 0)\end{array}$ & $\begin{array}{l}\text { BIG I-98b } \\
(\mathrm{N}=4922)\end{array}$ & $\begin{array}{l}\text { BIG I-98 } \\
(\mathrm{N}=4922)\end{array}$ \\
\hline Median follow-up & 68 months & 100 months & 25.8 months & 60.5 months & 51 months & 76 months \\
\hline HR for DFS & HR 0.83 & HR 0.85 & HR 0.81 & HR 0.86 & HR 0.82 & HR 0.88 \\
\hline$P$ value & $P=0.005$ & $P=0.003$ & $P=0.003$ & $P=0.008$ & $P=0.007$ & $P=0.03$ \\
\hline Risk reduction & $17 \%$ & $15 \%$ & $19 \%$ & $\begin{array}{l}\text { I4\% } \\
\text { CEN: HR } 0.83^{c} \\
17 \%^{c}\end{array}$ & $18 \%$ & $\begin{array}{l}12 \% \\
\text { CEN: HR } 0.84^{c} \\
16 \%\end{array}$ \\
\hline HR for TTDR & HR 0.84 & HR 0.84 & HR 0.73 & HR 0.79 & HR 0.8I & HR 0.85 \\
\hline$P$ value & $P=0.06$ & $P=0.022$ & $P=0.001$ & $P=0.003$ & $P=0.03$ & $P=0.05$ \\
\hline Risk reduction & $16 \%$ & $16 \%$ & $27 \%$ & $\begin{array}{l}21 \% \\
\text { CEN: HR 0.78c } \\
22 \%^{c}\end{array}$ & $19 \%$ & $\begin{array}{l}\text { I5\% } \\
\text { CEN: HR 0.8Ic } \\
19 \%^{c}\end{array}$ \\
\hline HR for OS & HR 0.97 & HR 0.97 & HR 0.86 & HR 0.87 & HR 0.9I & HR 0.87 \\
\hline$P$ value & $P=0.7$ & $P=0.7$ & $P=0.16$ & $P=0.07$ & $P=0.35$ & $P=0.08$ \\
\hline Risk reduction & $3 \%$ & $3 \%$ & $14 \%$ & $\begin{array}{l}13 \% \\
\text { CEN: HR 0.8I } \\
19 \%^{c}\end{array}$ & $9 \%$ & $\begin{array}{l}\text { I3\% } \\
\text { CEN: HR 0.8I } \\
19 \%\end{array}$ \\
\hline
\end{tabular}

Notes: a Results are for the hormone receptor-positive patient population of ATAC; ${ }^{\text {Results for monotherapy arms of BIG I-98; ' } E x c l u d e s ~} 619$ patients who crossed over to letrozole treatment arm after unblinding of tamoxifen treatment arm; HR and percent risk reduction are shown.

Abbreviations: ATAC, Arimidex Tamoxifen Alone or in Combination; BIG, Breast International Group; CEN, censored analysis; DFS, disease-free survival; HR, hazard ratio; OS, overall survival; TTDR, time to distant recurrence.

improved (Table 1). ${ }^{8}$ Distant DFS (DDFS) was not a defined endpoint in the ATAC trial; however, as reported in the package insert, the difference in DDFS events was 370 and 394 in the respective treatment groups in the HR+ population (HR 0.93; 95\% CI: $0.80-1.07$ ). ${ }^{18}$ As with the 33-month analysis, significant improvement in CLBC was observed in the HR+ population. There were 831 deaths in the study at this early follow-up, and the HR for OS was not statistically different in either the ITT (HR 0.97; 95\% CI: $0.85-1.12$ ) or HR+ (Table 1) populations, despite a $12 \%$ reduction in breast cancer-related deaths (HR 0.88 ; 95\% CI: $0.74-1.05 ; P=0.2){ }^{8,18}$

Results of the ATAC trial have been reported up to the 100-month ( 8.3 year) follow-up, at which time patients had received a mean of 4.11 and 3.97 years of anastrozole and tamoxifen treatment, respectively, with a high rate of compliance with randomized treatment $(88 \%$ and $87 \%$ of allocated treatment received before recurrence, respectively). ${ }^{7}$ In the $\mathrm{HR}+$ population, significant improvement was observed in the endpoints of DFS (Table 1), time to recurrence (TTR; HR 0.76; 95\% CI: $0.67-0.87 ; P=0.0001)$, and CLBC with anastrozole relative to tamoxifen (HR 0.60 ; $95 \%$ CI: $0.42-0.85$; $P=0.004) .{ }^{7}$ Of note, there were 52 fewer TTDR events with anastrozole relative to tamoxifen at this time point, and this reached significance in the HR+ population (305 vs 357, Table 1). All cause death (472 vs 477 events, Table 1), death after recurrence (245 vs 269 events, HR 0.90; $P=0.2$ ), and deaths without recurrence (227 vs 208 events, HR
$1.05 ; P=0.6)$ were not significantly different in the HR+ population.

\section{The Breast International Group (BIG) I-98 trial}

An important distinction between the BIG 1-98 trial and the ATAC trial is that BIG 1-98 was conducted in an independent manner by the International Breast Cancer Study Group, a large cooperative group responsible for study design, data collection, management, medical review, data analysis, and reporting. ${ }^{9}$ The unique design of BIG 1-98 allowed for the assessment of two adjuvant treatment strategies. The first compared initial adjuvant letrozole with tamoxifen for 5 years' total treatment, while the second compared the use of letrozole and tamoxifen, in either order, for a 2- and 3 -year treatment period, respectively, to complete a 5-year hormonal adjuvant treatment course in PMW with HR+ breast cancer. ${ }^{9}$ The Primary Core Analysis (PCA) compared all patients assigned to initial adjuvant letrozole $(n=4003)$ or tamoxifen ( $\mathrm{n}=4007)$, thus including patients assigned to sequential therapy for the first 2 years, on either letrozole or tamoxifen, while the monotherapy analysis compared only those patients who were assigned to the monotherapy (tamoxifen or letrozole) arms.

The ATAC and BIG 1-98 trials also differed significantly with respect to the definition of DFS. As noted earlier, ATAC defined a DFS event as local or distant recurrence, new primary breast cancer, or death from any cause, ${ }^{17}$ whereas 
BIG 1-98 included the occurrence of a second, nonbreast cancer in its definition and also added an additional endpoint of DFS excluding a second, nonbreast cancer, similar to ATAC. ${ }^{9}$ Median follow up for the PCA was 25.8 months (2.2 years); at this time, both DFS (as defined in BIG 1-98, Table 1) and DFS excluding a second, nonbreast cancer (HR 0.79; 95\% CI: 0.68-0.92; $P=0.002$ ) were significantly improved with letrozole relative to tamoxifen. ${ }^{9}$ TTR (HR 0.72 ; 95\% CI: $0.61-0.86 ; P<0.001$ ) also was significantly improved with letrozole, and importantly, TTDR at this early time point was improved by $27 \%$ with letrozole (184 vs 249 events, Table 1). There was no significant difference between the treatment groups in OS (166 vs 192 events, Table 1) at the time of this analysis.

In a subsequent, predefined analysis of BIG 1-98 in patients randomized to the monotherapy arms $(n=2463$ letrozole, $n=2459$ tamoxifen), median follow-up was approximately double that of the PCA, at 51 months (4.3 years). ${ }^{10}$ At this time, there were 352 DFS events in the letrozole arm, compared with 418 events in the tamoxifen arm (Table 1); TTR (231 vs 291 events, HR 0.78; 95\% CI: 0.65-0.92; $P=0.004)$ as well as TTDR (193 vs 234 events, Table 1) remained significantly improved with letrozole over tamoxifen in this analysis. ${ }^{10}$ Again, there were fewer overall deaths with letrozole versus tamoxifen (194 vs 211 events, Table 1), but the difference did not reach significance.

The pivotal results from the first PCA at 25.8 months led to a decision by the International Breast Cancer Study Group to unblind the tamoxifen arm of the trial (with all other arms remaining blinded), inform patients of the superiority of letrozole, and allow the selective crossover of patients from tamoxifen to letrozole treatment. ${ }^{11,12}$ In total, 619 patients, or $25.2 \%$ from the tamoxifen arm, elected to cross over to letrozole therapy, and most patients did so between years 3 and 5 of the trial. Of note, the crossover did not significantly affect results of the 51-month monotherapy analysis; however, subsequent comparisons between the letrozole and tamoxifen arms in the PCA and monotherapy populations, as detailed below, were affected.

In late 2008, extended follow-up for patients randomized to the monotherapy arms $(n=2463$ letrozole, $n=2459$ tamoxifen), at a median follow-up of 76 months (6.3 years), was reported. All patients had completed protocol treatment at the time of this predefined, 10-year analysis. ${ }^{11}$ In the ITT population, despite the above-noted crossover of about a quarter of patients, DFS was significantly improved in the letrozole arm (509 events) compared with the tamoxifen arm (565 events) (Table 1). Specifically, letrozole monotherapy resulted in fewer breast cancer events (321 vs 363) and fewer second, nonbreast malignancies (101 vs 115). Similar improvements within the letrozole arm occurred for TTDR compared with the tamoxifen arm (257 vs 298, Table 1). Importantly, there was a pronounced trend in favor of letrozole in OS compared with tamoxifen. There were 40 fewer deaths in the letrozole treatment arm versus the tamoxifen $\operatorname{arm}$ (303 vs 343, Table 1). In addition, the number of deaths without a prior cancer event was the same in both treatment groups ( 87 vs 87 ). As the crossover likely affected the comparison between the letrozole and tamoxifen groups, an exploratory analysis censoring events at the time of crossover was conducted. This analysis showed a greater and significant benefit of letrozole in all endpoints, including DFS, OS, and TTDR (Table 1). ${ }^{11}$ Additionally, an analysis called the inverse probability of censoring weighting was performed to handle bias created by censoring events and assess the true benefits of each treatment regimen. The results showed significant benefits with letrozole in DFS (HR 0.85; 95\% CI: 0.76-0.96) and OS (HR 0.83; 95\% CI: 0.71-0.97), with HRs residing between the results of the ITT and censored analyses. ${ }^{11}$ These data show an emerging survival benefit for patients treated with letrozole monotherapy over time.

Results presented in early 2009 also have detailed findings in the larger PCA population of BIG 1-98 $(\mathrm{N}=8010)$ at the predefined, 10-year update (median 60.5 month follow-up). ${ }^{12}$ As with the monotherapy analysis, despite the crossover of patients from tamoxifen to letrozole, there was a significant benefit of letrozole over tamoxifen in DFS (585 vs 664 events, Table 1), TTR (368 vs 441 events, HR 0.82; 95\% CI: 0.71-0.94; $P=0.004$ ), and TTDR (287 vs 357 events, Table 1), and a strong trend in OS favoring letrozole (330 vs 374 events, Table 1). In the exploratory censored analysis, the benefit of letrozole over tamoxifen was again greater and significant for all endpoints, including DFS, TTR, TTDR, and OS (Table 1). ${ }^{12}$ Results from the PCA population thus confirmed the findings of the monotherapy analysis, showing the emergence of a survival benefit over time with letrozole treatment.

\section{ATAC and BIG I-98: key efficacy differences}

Several important subgroups of breast cancer patients can be identified. One of the most important subgroups comprises patients with lymph node involvement (ie, node positive $[\mathrm{N}+])$, as they typically are more prone to early recurrence compared with node-negative (N0) patients (Figure 1). ${ }^{19,20}$ Results of both the PCA (HR 0.71; 95\% CI: 0.59-0.85; 


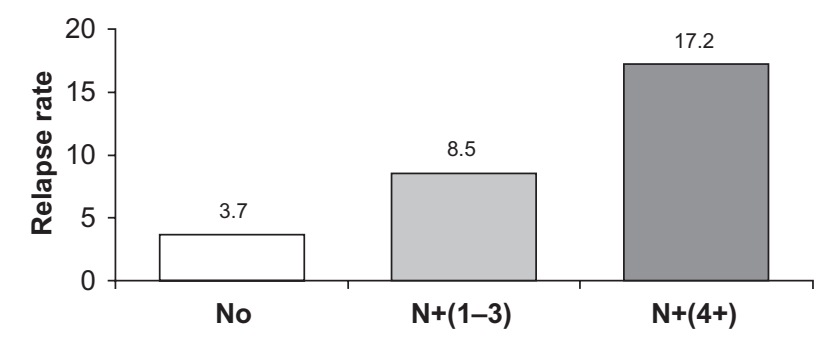

Figure I Increasing risk of early recurrence in patients with node-positive $(\mathrm{N}+)$ disease. The figure shows the relapse rate at 2.5 years for patients with increasing nodal involvement. Node-negative (N0) $(n=1962) ; \mathrm{N}+(\mathrm{I}-3)$, I to 3 involved nodes $(n=1650) ; N+(4+), 4$ or more involved nodes. ${ }^{17}$

$P<0.001)$ and the monotherapy analysis of BIG 1-98 (HR 0.77; 95\% CI: $0.64-0.92 ; P=0.004$ ) confirmed a significant benefit of letrozole over tamoxifen for DFS in the subgroup of $\mathrm{N}+$ patients $(\mathrm{n}=3311$ and $\mathrm{n}=2067$, respectively). There also was a significant benefit of letrozole for patients with prior chemotherapy (PCA: $\mathrm{n}=2024$, HR 0.70 ; $P=0.01$; monotherapy analysis: $\mathrm{n}=1232$, HR $0.74 ; P=0.03$ ) and in those with tumor size $>2 \mathrm{~cm}$ (PCA: $\mathrm{n}=2973$, HR $0.76 ; P=0.004$; monotherapy analysis: $\mathrm{n}=1858$, HR 0.79 ; $P=0.01) .{ }^{9,10}$ The updated results at 60.5 months from the PCA cohort also confirm a significant benefit of letrozole over tamoxifen in the subgroup of $\mathrm{N}+$ patients $(\mathrm{n}=3313)$ for both DFS (HR 0.79; 95\% CI: 0.68-0.91) and OS (HR 0.81 ; 95\% CI: 0.67-0.97). ${ }^{12}$ In the ATAC trial, the DFS benefit also favored anastrozole in these important high-risk patient subgroups $(\mathrm{N}+$ : HR 0.84 , tumors $>2 \mathrm{~cm}$ : HR 0.74 , prior chemotherapy: HR $0.89 ; P$ values were not reported at the 100-month analysis), and anastrozole benefits were maintained regardless of the use of prior chemotherapy or the type of chemotherapy. ${ }^{7,17,21}$

Comparison of the ATAC and BIG 1-98 trials also yields some important findings with respect to DM events, which have been associated with a greater risk of death overall as well as death due to breast cancer. ${ }^{22,23}$ In a retrospective analysis comparing the 33-month results from ATAC and the 25.8-month follow-up results from BIG 1-98 (as these first analyses represent the closest follow-ups between trials), DFS efficacy of these two drugs versus tamoxifen was similar, but DM events were significantly lower with letrozole (relative risk [RR] 0.76; 95\% CI: 0.63-0.92) compared with anastrozole (RR 0.87; 95\% CI: 0.70-1.06). ${ }^{24}$ In addition, a trend toward improved OS was observed with letrozole in this analysis (RR 0.87; 95\% CI: 0.71-1.06), arising primarily from a significant reduction in deaths after a cancer event (RR 0.72; 95\% CI: 0.57-0.92), whereas no such trend was observed in the ATAC trial. ${ }^{24} \mathrm{~A}$ retrospective analysis, focused specifically on early recurrence events occurring in
BIG 1-98 (at 2 years), found a 30\% reduction in early DM events with letrozole ( 87 vs 125 events, $2.3 \%$ vs 3.3\%). ${ }^{25,26}$ A similar unpublished retrospective analysis of early events in the ATAC trial revealed a comparatively smaller reduction in early DM events with anastrozole; distant recurrence at 2.5 years was reduced by $7 \%$ (133 events vs 143 events) with anastrozole relative to tamoxifen in the ITT population. ${ }^{26,27}$ A recently reported retrospective analysis of the $\mathrm{HR}+$ subgroup $(n=5216)$ in ATAC at 2 years showed a $21 \%$ relative reduction in DM events (HR 0.79; 95\% CI: 0.58-1.07) with anastrozole that was not significant, compared with a $32 \%$ relative reduction in overall recurrences, which was significant (HR 0.68; 95\% CI: 0.52-0.88). ${ }^{28}$ In an earlier report, Rugo et al used a number needed to treat (NNT) analysis to compare early recurrence results from ATAC and BIG 1-98. ${ }^{29}$ In this analysis, a lower NNT would indicate the more efficacious drug in preventing a given recurrence event (meaning fewer numbers of patients needing to be treated with that agent to prevent 1 recurrence). The results showed a major advantage of letrozole over anastrozole in the prevention of DM events (NNT $=100$ for letrozole vs 300 for anastrozole). By comparison, anastrozole showed a slight advantage in terms of local and regional recurrences $(\mathrm{NNT}=279$ vs 423) as well as CLBC (NNT = 156 vs 948), although it should be noted that the number of these latter recurrence events was far smaller at the early follow-up, and the study did not take into account recently reported early recurrence results from ATAC in the HR+ subgroup. ${ }^{28,29}$ Interestingly, both drugs were nearly identical for preventing DFS events (ie, recurrences overall), with an NNT of 77 for anastrozole versus 75 for letrozole.

Taken together, these findings suggest a meaningful benefit of letrozole over anastrozole in reducing early DM events, and the significance of this reduction should not be underestimated. In a recently published study $(\mathrm{N}=3614$ PMW with operable, estrogen receptor-positive breast cancer receiving adjuvant tamoxifen), a peak of recurrence was reported at 2 years ( $4.3 \%$ per annum), and DM events were found to be the predominant type of early recurrence event at this peak (3.4\% per annum). ${ }^{30}$ Retrospective analyses from the ATAC and BIG 1-98 trials also confirmed that DM events constitute the majority of recurrence events at 2 years..$^{25,28}$ Prevention of DM recurrence is thus a key factor in improving survival and reducing death from breast cancer. ${ }^{22,23}$

In light of the survival benefit with letrozole over tamoxifen, which has been seen in the later follow-ups of both the PCA and monotherapy cohorts, ${ }^{11,12}$ a recent study examined the NNT to save a life with anastrozole and letrozole. ${ }^{31}$ 
This analysis was based on data from the 68-month follow-up of ATAC ( $3 \%$ improvement in OS) and the 76-month follow up of the monotherapy arms of BIG 1-98 (censored analysis, $19 \%$ improvement in OS). The NNT to avoid one early distant recurrence was significantly lower with letrozole versus anastrozole (100 vs 303, threefold difference), as was the NNT required to avoid one death (63 vs 161, 2.5-fold difference). The investigators speculated that the difference in the reduction of early DM events with letrozole versus anastrozole could account for the latter difference in deaths. ${ }^{31}$ It also should be noted that the magnitude of the survival benefit seen with letrozole over tamoxifen, as expressed in terms of NNT, is comparable with that seen with other paradigmchanging adjuvant regimens (eg, the addition of taxanes to anthracycline-based chemotherapy regimens). ${ }^{32}$

\section{Binding and potency of anastrozole and letrozole}

The potency of AIs relates to their ability to specifically suppress the enzyme aromatase, and in this regard, the third-generation AIs including letrozole and anastrozole demonstrate clear advantages over first-generation compounds such as aminoglutethimide. In an early, open-label, randomized, comparative trial of letrozole $(\mathrm{n}=185,2.5 \mathrm{mg}$ letrozole; $\mathrm{n}=192,0.5 \mathrm{mg}$ letrozole) with aminoglutethimide $(\mathrm{n}=178)$ in women with advanced breast cancer, letrozole at 2.5 or $0.5 \mathrm{mg}$ resulted in an objective response rate (ORR) of $19.5 \%$ and $16.7 \%$, respectively, versus only $12.4 \%$ for aminoglutethimide, and there was a significantly better time to progression (RR $0.72 ; 95 \% \mathrm{CI}: 0.57-0.92 ; P=0.008$ ), time to treatment failure (RR 0.70; 95\% CI: $0.55-0.88 ; P=0.003$ ), and OS (RR 0.64; 95\% CI: $0.49-0.85 ; P=0.002$ ) for letrozole $2.5 \mathrm{mg}$ versus aminoglutethimide. ${ }^{33}$ Another study of advanced breast cancer patients compared letrozole $(n=356)$ with anastrozole $(n=357)$ as second-line therapy. ${ }^{34}$ In this study, median time to progression, the primary endpoint, was identical for the two drugs (5.7 months). However, ORR was significantly improved with letrozole relative to anastrozole (19.1\% vs $12.3 \%, P=0.013) .{ }^{34}$ It is unclear whether these differences in clinical efficacy are directly correlated with the degree of aromatase inhibition and the corresponding suppression of plasma $\mathrm{E}_{2}$; however, it is notable that the potency of aminoglutethimide in this regard (90.6\% suppression) is substantively lower than that of letrozole (nearly 99\% suppression). ${ }^{6,35,36}$

Although both anastrozole and letrozole are nonsteroidal AIs, there are important differences between these two compounds, which could, in part, underlie the clinical results that have been observed. Computer-assisted molecular modeling studies suggest that letrozole fits very tightly into the binding site of the aromatase enzyme and occupies the entire binding site, whereas anastrozole does not (Figure 2). ${ }^{4,37}$ In a recent study, plasma samples from a neoadjuvant study using letrozole (16 weeks' neoadjuvant treatment followed by surgery) were directly compared with samples from a similar neoadjuvant trial of anastrozole using a highly sensitive assay for $\mathrm{E}_{2}$ detection. ${ }^{15}$ The authors found a significant difference in the degree of plasma $\mathrm{E}_{2}$ suppression for letrozole versus anastrozole ( $95.2 \%$ vs $92.8 \%, P=0.018)$, and there were similar, significant differences observed for estrone $(98.8 \%$ vs $96.3 \%, P=0.003)$ and estrone sulfate (98.9\% vs $95.3 \%, P=0.003) .{ }^{15}$ The suppression of plasma $\mathrm{E}_{2}$ by these two AIs also has been directly compared in the ALIQUOT (Anastrozole versus Letrozole, an Investigation of Quality Of Life and Tolerability) trial. This study compared outcomes following 3 months of adjuvant treatment with letrozole or anastrozole in a randomized crossover design in 54 PMW with HR+ breast cancer. ${ }^{16}$ Patients were randomized to letrozole for 12 weeks followed by anastrozole for 12 weeks $(n=27)$, or the reverse sequence $(n=27)$, and plasma levels of $\mathrm{E}_{2}$ were assayed following each drug treatment (Figure 3a). Significantly fewer patients had $\mathrm{E}_{2}$ plasma values of $3 \mathrm{pmol} / \mathrm{L}$ or greater (Figure $3 \mathrm{~b}$ ), the percent residual $\mathrm{E}_{2}$ level was lower (Figure $3 \mathrm{c}$ ), and the mean plasma $\mathrm{E}_{2}$ level after extrapolation was significantly lower following letrozole versus anastrozole treatment (Figure 3d). ${ }^{16}$ These results were consistent regardless of which agent was given first in the study and regardless of prior tamoxifen use. Sup-
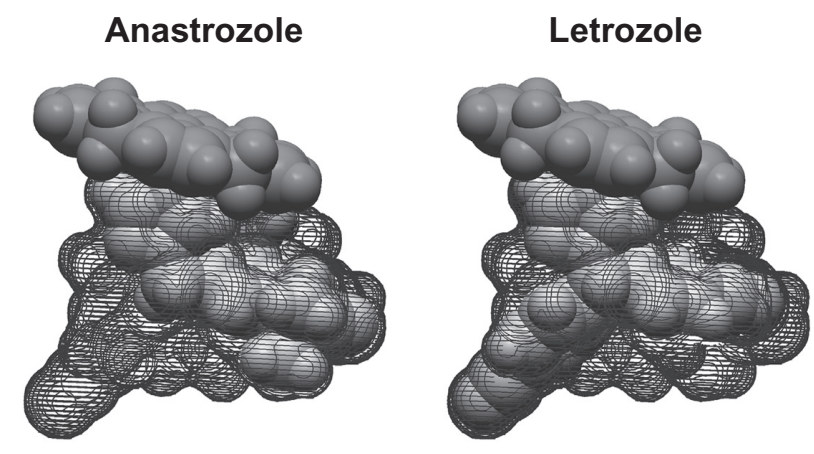

Figure 2 Binding of nonsteroidal aromatase inhibitors anastrozole and letrozole. Computer-assisted molecular modeling displaying the active site fit and heme group binding in the aromatase enzyme. Whereas the distal portion of the letrozole molecule fits tightly and occupies the entire binding site, the same is not true for anastrozole. ${ }^{4,37}$ Reprinted with permission from Mouridsen HT, Bhatnagar AS. Letrozole in the treatment of breast cancer. Expert Opin Pharmacother. 2005;6:1389-1399. ${ }^{4}$ Copyright @ 2005, Ashley Publications Ltd. 

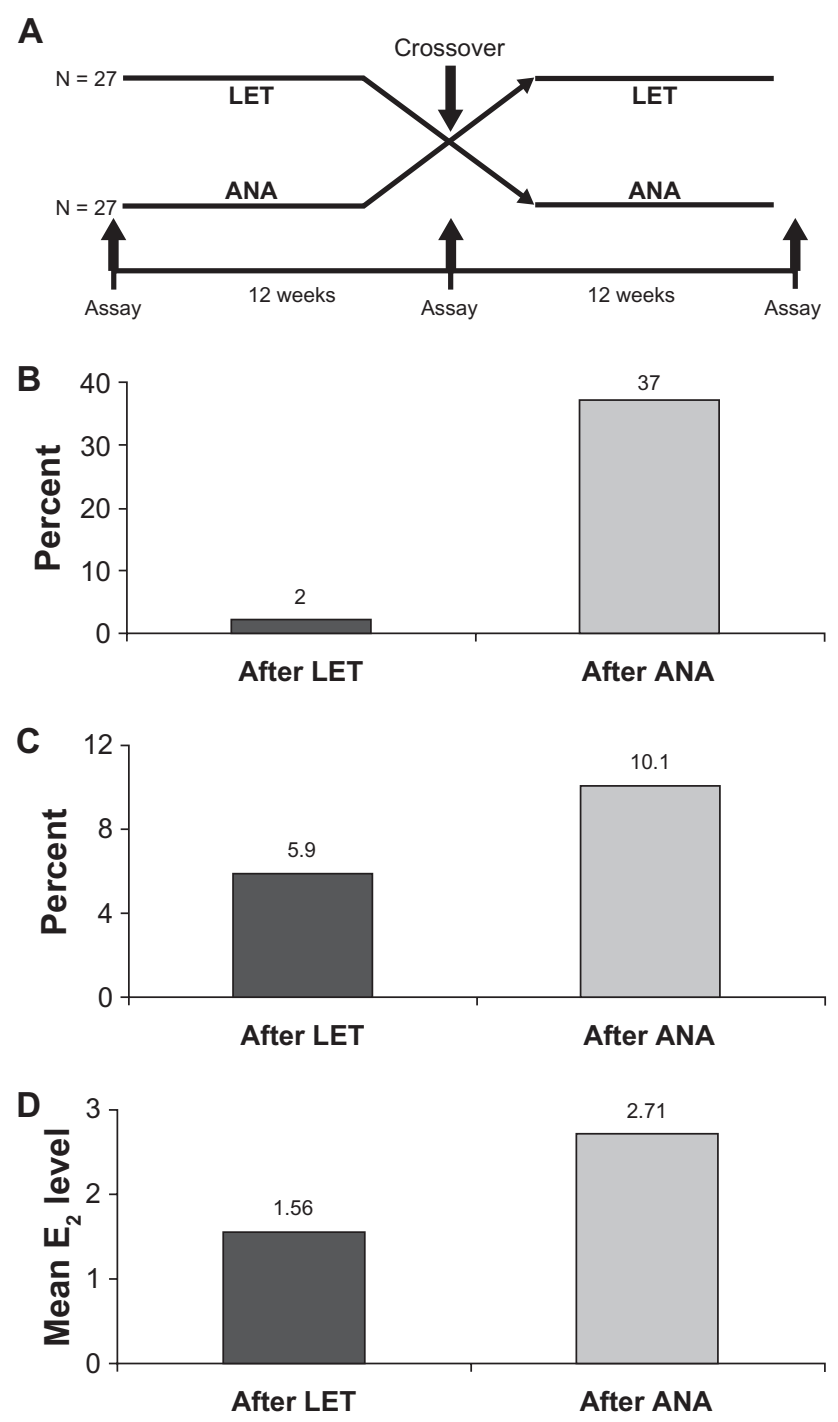

Figure 3 A) Study design of ALIQUOT (Anastrozole versus Letrozole, an Investigation of Quality Of Life and Tolerability), an open-label, crossover study comparing adjuvant therapy with letrozole and anastrozole. Postmenopausal women with hormone receptor-positive breast cancer $(n=27$ per group) were randomized to 3 months' treatment with letrozole (LET) followed by anastrozole (ANA), or the reverse sequence; plasma estrogen $\left(E_{2}\right)$ levels were assayed at the indicated time points. B) Percent of patients with an $E_{2}$ value of $3 \mathrm{pmol} / \mathrm{L}$ or greater following treatment with LET or ANA; the difference was statistically significant. C) Mean residual $E_{2}$ level (\%) following treatment with LET or ANA. D) Mean $E_{2}$ level in $\mathrm{pmol} / \mathrm{L}$ after extrapolation following treatment with either LET or ANA; the difference was statistically significant. ${ }^{16}$

pression of estrogen in the breast tumor tissue also has been found to be more pronounced following neoadjuvant letrozole compared with anastrozole. Mean percentages of suppression with letrozole and anastrozole were $97.6 \%$ and $89.0 \%$ for $\mathrm{E}_{2}, 90.7 \%$ vs $83.4 \%$ for estrone, and $90.1 \%$ vs $72.9 \%$ for estrone sulfate, respectively. ${ }^{15}$ Collectively, these findings provide evidence for a greater inhibition of aromatase and a greater corresponding suppression of plasma and tissue $\mathrm{E}_{2}$ levels with letrozole relative to anastrozole, and a possible explanation for their differences in the clinical setting. It is quite plausible that lower plasma $\mathrm{E}_{2}$ levels afforded by letrozole treatment provide for more complete eradication of micrometastatic tumor deposits, which could in turn result in fewer DM recurrences. ${ }^{26}$

\section{FACE: trial design and methods}

As outlined above, there is evidence from clinical and pharmacodynamic data to suggest a difference in potency between the nonsteroidal AIs anastrozole and letrozole. Nevertheless, the clinical data presented herein admittedly are from different trials (ATAC and BIG 1-98) that were conducted at different times and with different overall goals, and with substantive differences in study design, population, and follow-up reporting of results. Accordingly, only limited conclusions can be drawn from such indirect comparisons. More meaningful comparisons between agents ideally should come from head-to-head trails. The FACE trial was designed with the caveats of cross-trial comparisons in mind, and forthcoming results will begin to address the important issue of efficacy and safety differences between the thirdgeneration AIs. FACE was designed as a head-to-head, Phase III, open-label, randomized, multicenter (up to 250 international sites) clinical trial comparing adjuvant treatment with anastrozole (1 mg daily) or letrozole ( $2.5 \mathrm{mg})$ in more than 4000 PMW with HR+ and N+ breast cancer. ${ }^{38,39}$ As of March 2008, accrual into the trial was completed, with 4172 patients enrolled. Consequently, safety and efficacy interim data are imminent. Patients who were $\mathrm{N}+$ were specifically chosen for FACE, as these women are known to be at a higher overall relapse risk $^{19,20}$ and are prone to earlier recurrence (within 2 years). ${ }^{25}$ These women would therefore benefit the most from initial adjuvant therapy with an AI, as opposed to initial tamoxifen therapy. In addition, such a patient population could theoretically allow for a statistical difference in efficacy between these AIs to emerge in a shorter period of time. Treatment was to commence following standard chemotherapy or radiotherapy (if given), and patients were to receive treatment for up to 5 years, or until disease recurrence/relapse. Patients were to be stratified according to the number of involved nodes, as well as human epidermal growth factor receptor 2 status. ${ }^{38,39}$

The primary endpoint of FACE is DFS at 5 years for letrozole and anastrozole, with the important additional secondary endpoints of OS, TTDR, time to CLBC, and safety. The safety results also will be particularly important to determine any potential differences between these 
AIs with respect to bone health and cardiovascular adverse events. The trial is sufficiently powered to detect a difference of $3.5 \%$ between the two treatment arms at 5 years (ie, 5 years' DFS of $76.5 \%$ for anastrozole vs $80.0 \%$ for letrozole); this corresponds to an HR of 0.83 in favor of letrozole. Interim efficacy analyses for DFS also are planned when approximately a third of the maximum number of events have occurred, and again when about two-thirds of the maximum events have occurred (about 320 and 639 events, respectively). Finally, a number of correlative science studies will be undertaken in FACE. These include an assessment of $\mathrm{E}_{2}$ suppression during treatment with anastrozole or letrozole, and the use of exploratory proteomics and genomics studies to identify differential protein, blood metabolite, and/or gene expression profiles, if any, that are associated with differential DFS and OS with anastrozole or letrozole therapy. As with BIG 1-98, the data will be provided by an independent academic organization, the Michelangelo Foundation, and will be reviewed in a confidential manner by an Independent Data Monitoring Committee (IDMC) chaired by Dr H Rugo; the IDMC will make recommendations to the Trial Steering Committee, co-chaired by Professor I Smith and Dr J O'Shaughnessy. The Steering Committee has developed a publication plan, and will determine when abstracts and/or manuscripts originating from the trial will be developed and presented. At annual intervals, and at the time of the interim analyses, the validated database will be transferred to the Michelangelo Foundation, which will work closely with the IDMC to monitor the study. Only after the final analysis has been completed will the database be transferred to the study sponsor, Novartis.

\section{Conclusions}

Evidence from the pivotal trials of anastrozole and letrozole, ATAC and BIG 1-98, has revealed important efficacy differences between these two drugs (Table 1), particularly in the prevention of early DM events, which are by far the most prevalent and the most deadly types of breast cancer recurrence events. ${ }^{22,23,25,27,28,30}$ Differences in the binding of letrozole and anastrozole to the aromatase enzyme (Figure 1), and their corresponding inhibitory activity, may in part underlie the more complete suppression of $\mathrm{E}_{2}$ levels with letrozole versus anastrozole that has been observed in directly comparative clinical studies such as ALIQUOT (Figure 3). It is plausible that the more complete suppression of $\mathrm{E}_{2}$ in plasma and tissues by letrozole in turn causes a greater eradication of distant micrometastatic breast cancer deposits, which can develop later into fulminant DM recurrences. This especially may be relevant for patients with $\mathrm{N}+$ disease, as these patients present a greater risk for early recurrence (Figure 2). Although letrozole effectively suppresses recurrence in all relevant patient subgroups, its benefit is especially pronounced in the high-risk subgroup of $\mathrm{N}+$ patients. ${ }^{9,10}$ In addition, while not reported in the 76-month follow-up of the monotherapy arms, subgroup analysis in the PCA population at 60.5 months shows a significant DFS and OS benefit in the $\mathrm{N}+$ subgroup, despite the crossover of about $25 \%$ of patients from tamoxifen to letrozole. ${ }^{12}$

There is precedent for an association between a lower degree of aromatase inhibition (ie, in the case of aminoglutethimide) and poorer outcomes compared with letrozole in advanced cancer patients. ${ }^{33}$ Despite these observations, however, comparisons with anastrozole remain indirect, and only directly comparative head-to-head trials will be useful in resolving the question of potential efficacy differences between letrozole and anastrozole. The development and design of the FACE trial undoubtedly will provide a conclusive resolution to this question, and demonstrate unequivocally which AI is more appropriately used upfront in the high-risk population of $\mathrm{N}+$ breast cancer patients. It is hoped that other similarly designed trials will be implemented to enable further comparisons among the third-generation AIs in different patient populations and treatment settings. In this regard, the National Cancer Institute of Canada MA. 27 trial is investigating upfront anastrozole versus exemestane in the adjuvant setting in PMW with HR+ breast cancer. ${ }^{40}$ Analysis of the trial is forthcoming; to date, 6830 patients have been accrued. Until the results of this trial and the FACE trial are reported, however, clinicians need to rely on the available clinical evidence, which strongly favors the use of upfront letrozole for PMW with HR+ early breast cancer.

\section{Acknowledgment}

Financial support for medical editorial assistance was provided by Novartis Pharmaceuticals. We thank Maria Soushko, PhD, of Phase Five Communications Inc., for medical editorial assistance with this manuscript.

\section{Disclosure}

The author discloses no conflicts of interest.

\section{References}

1. Early Breast Cancer Trialists' Collaborative Group (EBCTCG). Effects of chemotherapy and hormonal therapy for early breast cancer on recurrence and 15-year survival: an overview of the randomised trials. Lancet. 2005;365:1687-1717. 
2. Fisher B, Costantino JP, Wickerham DL, et al. Tamoxifen for prevention of breast cancer: report of the National Surgical Adjuvant Breast and Bowel Project P-1 Study. J Natl Cancer Inst. 1998;90:1371-1388.

3. Braithwaite RS, Chlebowski RT, Lau J, George S, Hess R, Col NF. Meta-analysis of vascular and neoplastic events associated with tamoxifen. J Gen Intern Med. 2003;18:937-947.

4. Mouridsen HT, Bhatnagar AS. Letrozole in the treatment of breast cancer. Expert Opin Pharmacother. 2005;6:1389-1399.

5. Rieber AG, Theriault RL. Aromatase inhibitors in postmenopausal breast cancer patients. J Natl Compr Canc Netw. 2005;3:309-314.

6. Geisler J, Lonning PE. Aromatase inhibition: translation into a successful therapeutic approach. Clin Cancer Res. 2005;11:2809-2821.

7. Arimidex, Tamoxifen, Alone or in Combination (ATAC) Trialists' Group; Forbes JF, Cuzick J, Buzdar A, Howell A, Tobias JS, Baum M. Effect of anastrozole and tamoxifen as adjuvant treatment for early-stage breast cancer: 100-month analysis of the ATAC trial. Lancet Oncol. 2008;9:45-53.

8. Howell A, Cuzick J, Baum M, et al; ATAC Trialists' Group. Results of the ATAC (Arimidex, Tamoxifen, Alone or in Combination) trial after completion of 5 years' adjuvant treatment for breast cancer. Lancet 2005;365:60-62.

9. Breast International Group (BIG) 1-98 Collaborative Group; Thürlimann B, Keshaviah A, Coates AS, et al. A comparison of letrozole and tamoxifen in postmenopausal women with early breast cancer. $N$ Engl J Med. 2005;353:2747-2757.

10. Coates AS, Keshaviah A, Thürlimann B, et al. Five years of letrozole compared with tamoxifen as initial adjuvant therapy for postmenopausal women with endocrine-responsive early breast cancer: update of study BIG 1-98. J Clin Oncol. 2007;25:486-492.

11. BIG 1-98 Collaborative Group; Mouridsen H, Giobbie-Hurder A, Goldhirsch A, et al. Letrozole therapy alone or in sequence with tamoxifen in women with breast cancer. $N$ Engl J Med. 2009;361: 766-776.

12. Thurlimann B; BIG 1-98 Collaborative and International Breast Cancer Study Groups. Letrozole versus tamoxifen as adjuvant endocrine therapy for postmenopausal women with receptor-positive breast cancer. 39th St. Gallen Symposium; 2009 May 7-9; St. Gallen, Switzerland. Abstr 0161 .

13. Coombes RC, Kilburn LS, Snowdon CF, et al; Intergroup Exemestane Study. Survival and safety of exemestane versus tamoxifen after 2-3 years' tamoxifen treatment (Intergroup Exemestane Study): a randomised controlled trial. Lancet. 2007;369:559-570.

14. Goss, PE, Ingle JN, Martino S, et al. Randomized trial of letrozole following tamoxifen as extended adjuvant therapy in receptor-positive breast cancer: updated findings from NCIC CTG MA.17. J Natl Cancer Inst. 2005;97:1262-1271.

15. Geisler J, Helle H, Ekse D, et al. Letrozole is superior to anastrozole suppressing breast cancer tissue and plasma estrogen levels. Clin Cancer Res. 2008;14:6330-6335.

16. Dixon JM, Renshaw L, Young O, et al. Letrozole suppresses plasma estradiol and estrone sulphate more completely than anastrozole in postmenopausal women with breast cancer. J Clin Oncol. 2008;26: 1671-1676.

17. Baum M, Buzdar AU, Cuzick J, et al; for the ATAC Trialists Group. Anastrozole alone or in combination with tamoxifen versus tamoxifen alone for adjuvant treatment of postmenopausal women with early breast cancer: first results of the ATAC randomized trial. Lancet. 2002;359:2131-2139. Erratum in: Lancet. 2002;360:1520.

18. Arimidex [package insert]. Wilmington, DE: AstraZeneca; Rev. $12 / 08$.

19. McArthur HL, Olivotto I, Gelmon KA, et al. Risk of early relapse in post-menopausal women with early stage, estrogen receptor positive $(\mathrm{ER}+)$ breast cancer on tamoxifen. Breast Cancer Res Treat. 2005; 94 Suppl 1:S124. Abstr 3001.

20. Hortobagyi GN, Kau S-W, Buzdar AU, et al. What is the prognosis of patients with operable breast cancer (BC) five years after diagnosis? Proc Am Soc Clin Oncol. 2004;23:23. Abstr 585.
21. Buzdar AU, Guastalla JP, Nabholtz JM, Cuzick J; ATAC Trialists' Group. Impact of chemotherapy regimens prior to endocrine therapy: Results from the ATAC (Anastrozole and Tamoxifen, Alone or in Combination) trial. Cancer. 2006;107:472-480.

22. Lamerato L, Havstad S, Gandhi S, Jones D, Chlebowski R. Breast cancer recurrence and related mortality in US pts with early breast cancer. J Clin Oncol. 2005;23(16S):62s. Abstr 738.

23. Doughty JC, Mansell J, Monypenny I, et al. Distant recurrence and subsequent mortality in breast cancer patients treated at 5 UK centres. 39th St. Gallen Symposium; 2009 May 7-9; St. Gallen, Switzerland. Abstr 0188.

24. Vakaet L, Buyse M. Comparative results of two adjuvant trials comparing 5 years of an aromatase inhibitor (AI) with 5 years of tamoxifen (TAM) in postmenopausal women with early breast cancer (EBC). Breast Cancer Res Treat. 2006;100 Suppl 1:S108-S109. Abstr 2084.

25. Mauriac L, Keshaviah A, Debled M, et al; BIG 1-98 Collaborative Group; International Breast Cancer Study Group. Predictors of early relapse in postmenopausal women with hormone receptor-positive breast cancer in the BIG 1-98 trial. Ann Oncol. 2007;18:859-867.

26. Gligorov J, Azria D, Pivot X, Penault-Llorca F, Spielmann M, Namer M. Clinical impact of upfront adjuvant AI therapy on the early risk of recurrence. Eur J Cancer. 2007;5(Suppl):209. Abstr 2089.

27. Houghton J, on behalf of the ATAC Trialists' Group. Initial adjuvant therapy with anastrozole $(\mathrm{S})$ reduced rates of early breast cancer recurrent and adverse events compared with tamoxifen $(\mathrm{T})$ - data reported on behalf of the ATAC ('Arimidex', TAM, Alone or in Combination) Trialists’ group. Ann Oncol. 2006;17 Suppl 9:ix93. Abstr 243PD.

28. Howell A, Forbes J, Cuzick J. Initial adjuvant therapy with anastrozole - early and late event data from the Arimidex, Tamoxifen, Alone or in Combination (ATAC) trial in the hormone-responsive population. 39th St. Gallen Symposium; 2009 May 7-9; St. Gallen, Switzerland. Abstr 0130.

29. Rugo H, Rourke M, Dranitsaris G, Kaura S. Letrozole or anastrozole for the prevention of early recurrences in postmenopausal women with early stage breast cancer: Using number needed to treat (NNT) to compare benefit. Eur J Cancer Suppl. 2008;6:118. Abstr 236.

30. Mansell J, Monypenny IJ, Skene AI, et al. Patterns and predictors of early recurrence in postmenopausal women with estrogen receptorpositive early breast cancer. Breast Cancer Res Treat. 2009;117: 91-98.

31. Rugo HS, Kaura S, Dranitsaris G. The number needed to treat to save a life: a comparison between aromatase inhibitors. 39th St. Gallen Symposium; 2009 May 7-9; St. Gallen, Switzerland. Poster 136.

32. Dhillon N, Kaura S, Dranitsaris G, Rugo H. Number needed to treat (NNT) to compare benefits of letrozole (LET) with adjuvant chemotherapy (CT) in patients (pts) with node-positive $(\mathrm{N}+)$ breast cancer (BC). J Clin Oncol. 2009;27:15s. Abstr 581.

33. Gershanovich M, Chaudri HA, Campos D, et al. Letrozole, a new oral aromatase inhibitor: Randomised trial comparing $2.5 \mathrm{mg}$ daily, $0.5 \mathrm{mg}$ daily and aminoglutethimide in postmenopausal women with advanced breast cancer. Ann Oncol. 1998;9:639-645.

34. Rose C, Vtoraya O, Pluzanska A, et al. An open randomized trial of second-line endocrine therapy in advanced breast cancer. Comparison of the aromatase inhibitors letrozole and anastrozole. Eur J Cancer. 2003;39:2318-2327

35. Dowsett M, Jones A, Johnston SR, Jacobs S, Trunet P, Smith IE. In vivo measurement of aromatase inhibition by letrozole (CGS 20267) in postmenopausal patients with breast cancer. Clin Cancer Res. 1995;1:1511-1515.

36. MacNeill FA, Jones AL, Jacobs S, Lønning PE, Powles TJ, Dowsett M. The influence of aminoglutethimide and its analogue rogletimide on peripheral aromatisation in breast cancer. Br J Cancer. 1992;66: 692-697.

37. Furet P, Batzl C, Bhatnagar A, Francotte E, Rihs G, Lang M. Aromatase inhibitors: synthesis, biological activity, and binding mode of azole-type compounds. J Med Chem. 1993;36:1393-1400. 
38. Monnier A. Refining the postmenopausal breast cancer treatment paradigm: the FACE trial. Expert Rev Anticancer Ther. 2006;6: 1355-1359.

39. de Boer R Sr, Burris HA, Monnier A, et al; on behalf of the $\mathrm{H} 2 \mathrm{H}$ trial steering committee. The Head to Head trial: Letrozole vs anastrozole as adjuvant treatment of postmenopausal patients with node positive breast cancer. J Clin Oncol. 2006;24(18s):582s. Abstr 10672.
40. NCIC Clinical Trials Group. Exemestane or anastrozole in treating postmenopausal women who have undergone surgery for primary breast cancer. http://clinicaltrials.gov/ct2/show/NCT00066573. Accessed 2010 Jun 7.

\section{Publish your work in this journal}

Cancer Management and Research is an international, peer-reviewed open access journal focusing on cancer research and the optimal use of preventative and integrated treatment interventions to achieve improved outcomes, enhanced survival and quality of life for the cancer patient. The journal welcomes original research, clinical \& epidemiological studies, reviews \& evaluations, guidelines, expert opinion \& commentary, case reports \& extended reports. The manuscript management system is completely online and includes a very quick and fair peerreview system, which is all easy to use. Visit http://www.dovepress.com/ testimonials.php to read real quotes from published authors.

Submit your manuscript here: http://www.dovepress.com/cancer-management-and-research-journal 